\title{
Paracoccidioidomicose em crianças em Belém do Pará
}

\author{
Paracoccidioidomycosis in children in Belém, Pará State, Brazil
}

Eliane R.S. Fonseca, Pedro P.O. Pardal e Luiz Carlos Severo

\begin{abstract}
Resumo No período janeiro de 1985 e julho de 1996 foram observados 102 casos de paracoccidioidomicose em hospital de Belém, PA. Treze pacientes eram crianças entre 3 e 13 anos de idade, com predomínio do sexo feminino (8:5). Todos apresentavam a forma disseminada subaguda da micose. E comentada a alta prevalência da micose em crianças em áreas endêmicas da Região Amazônica.
\end{abstract}

Palavras-chaves: Paracoccidioidomicose (PCM) na infância. PCM do tipo juvenil. PCM subaguda.

\begin{abstract}
During the period from January/85 to July/96, 102 cases of paracoccidioidomycosis were observed in a hospital in Belém, PA. Thirteen of these cases were children between 3 and 13 years of age, with a predominance of females (8:5). All patients presented the disseminated subacute form of the mycosis. The high prevalence of the mycosis in children in endemic areas of the Amazon region is discussed.
\end{abstract}

Key-words: Paracoccidioidomycosis (PCM) in childhood. Juvenil type PCM. Subacute PCM.

Em 1950, Guimarães e Macedo6 relataram um caso de paracoccidioidomicose (PCM) em paciente paraense. Em 1954, Azevedo2 refere sumariamente outros seis casos. Em 1988, Naiff et al9 informam da ocorrência de 13 casos da micose, no espaço de dois anos e meio, em hospital de uma vila a margem da transamazônica; entre esses casos, dois em crianças nativas. Em 1995, Veras ${ }^{12}$, revisando os prontuários de 286 pacientes com PCM, internados em Teresina
(PI), no período 1980-1994, verifica que 91 $(31,8 \%)$ deles procediam do sudeste do Pará e, desses 23 (8\%) eram nativos do Estado. Esses dados mostram que no mundo amazônico há importantes áreas endêmicas de PCM.

O presente trabalho tem por objetivo acrescentar dados que suportam essa assertiva, destacando a importância de estudos epidemiológicos e ecológicos, para aquilatar a magnitude do problema.

\section{MATERIAL E MÉTODOS}

Foram revistos os prontuários de 102 pacientes com PCM atendidos no Hospital Universitário João de Barros Barreto (HUJBB), em Belém, PA, no período de janeiro de 1988 a

julho de 1996. Treze desses doentes eram crianças com idades entre 3 e 13 anos.

O diagnóstico da micose foi fundamentado no achado de formas características de

Doenças Infecciosas e Parasitárias, Hospital Universitário João de Barros Barreto, Universidade Federal do Pará, Belém, PA. Endereço para correspondência: Dr. Luiz C. Severo, Laboratório de Microbiologia Clínica, IPD/Santa Casa. Annes Dias 285, $90020-090$ Porto Alegre, RS, Brasil 
Paracoccidioides brasiliensis ao exame microscópico de especime clínico montado em solução de potassa a $20 \%$ ou de cortes histológicos de material obtido por biópsia. Em alguns casos o diagnóstico foi complementado pelo isolamento do fungo em cultivo.

\section{RESULTADOS}

No período de janeiro de 1985 a julho de 1996, foram atendidos no HUJBB 102 pacientes com PCM. Treze (12,7\%) deles eram crianças com idades entre 3 e 13 anos.

Doze pacientes procediam de municípios do Estado do Pará: Altamira 5 casos; Parauapebas 2 casos; e, Redenção, São Félix do Xingu, Paragominas, Itaituba e Xinguara, 1 caso cada. $O$ paciente restante procedia de Esperantina, Estado de Tocantins, município limítrofe. Estas crianças eram brancas, nativas do local, procedentes de região de desmatamento; todas apresentavam desnutrição protéico-calórica.

Sete dos pacientes tinham idades entre 3 e 10 anos, os outros seis entre 11 e 13 anos. Oito pacientes eram meninas, cinco eram meninos. Todos os pacientes apresentavam a forma disseminada subaguda (tipo juvenil) da PCM. O tempo de evolução da doença até o diagnóstico variou de 1 a 7 meses.

Os sintomas mais freqüentes foram: febre em 12 (92,3\%) pacientes; emagrecimento em
11 (84,6\%); aumento de volume do abdômen 6 (46\%) e, tosse, sem expectoração, 5 (38,5\%).

Os achados ao exame físico foram: adenomegalias superficiais em $100 \%$ dos pacientes (cadeias cervicais em 13 doentes; inguinal em 8 , submandibular em 5 , retroauricular em 3, supraclavicular em 1 e submentoniana em 1); hepatomegalia em 6 pacientes; esplenomegalia em 3 pacientes; massas intraabdominais em 3 pacientes; ascite em 3 pacientes; lesões cutâneas em 2 pacientes; lesões ósseas em 1 paciente; e, icterícia em 1 paciente.

O hemograma revelou anemia em todos os pacientes, leucositose em 6 , leucopenia em 1 , linfopenia em 9 e eosinofilia em 3.

O radiograma torácico evidenciou: alargamento do mediastino em 7 doentes; infiltrado em ambos os lobos inferiores de um menino de 8 anos; derrame pleural extenso em menina de 13 anos. Em cinco pacientes não foi evidenciada alteração radiológica. Uma menina de 3 anos de idade apresentou lesões osteolíticas difusas nos arcos costais, cintura escapular, tíbia e crista ilíaca.

\section{DISCUSSÃO}

O isolamento de Paracoccidioides brasiliensis de vísceras de tatus (Dasypus novemcinctus) no Pará demonstra que o fungo é indígena na Região Amazônica10. Em 1971, Campbell3 predisse que a medida que o homem avançasse, disturbando o solo, em regiões onde o fungo é nativo, a PCM constituiria uma ameaça cada vez maior. Tal está acontecendo em vastas áreas dos Estados do Pará, Maranhão e Tocantins, colonizados nas três últimas décadas.

PCM que ocorre nesses Estados apresenta uma peculiaridade interessante: a alta prevalência de casos em crianças. Esses casos comparecem nas casuísticas em percentuais dois a três vezes maiores que os observados em outras regiões endêmicas do Brasil (Tabela 1). Porque a criança tem suas atividades em áreas limitadas e as famílias não migram, Veras 12 utilizou seus casos infantis para apontar reservareas de PCM. Por isso, é de presumir-se que crianças de baixa idade com PCM possam ser ótimos indicadores de áreas menores onde a infecção tenha sido adquirida.

É provável que um maior interesse pela PCM da criança, especialmente na região Norte, onde é freqüente, venha demonstrar que nessa região a micose na sua forma disseminada

Tabela 1 - Prevalência de paracoccidioidomicose disseminada subaguda, em crianças, em casuísticas de algumas regiões do Brasil

\begin{tabular}{|c|c|c|c|c|c|c|}
\hline \multirow{3}{*}{ Região } & \multirow{3}{*}{ Estado } & \multirow{3}{*}{ Período } & \multicolumn{3}{|c|}{ Casos de paracoccidioidomicose } & \multirow{3}{*}{ Ref. } \\
\hline & & & \multicolumn{2}{|c|}{ Em crianças } & \multirow[t]{2}{*}{ Total } & \\
\hline & & & $\mathrm{n}^{\circ}$ & $\%$ & & \\
\hline \multirow[t]{2}{*}{ Norte } & PA, TO, MA & $1980-94$ & 41 & 14,3 & 286 & 12 \\
\hline & PA, TO & $1988-96$ & 13 & 12,7 & 102 & * \\
\hline \multirow[t]{2}{*}{ Sudeste } & MG & $1982-87$ & 3 & 4,7 & 64 & 5 \\
\hline & $\mathrm{SP}$ & $1944-74$ & 70 & 3,9 & 1890 & 4 \\
\hline Centro-Oeste & GO & $1965-80$ & 11 & 5,6 & 195 & 1 \\
\hline Sul & PR & $1962-85$ & 25 & 4,0 & 616 & 7 \\
\hline
\end{tabular}


subaguda da criança possa apresentar-se por quadros clínicos multiformes, como ocorre em outras regiões do país9.

Na região Norte é provável, também, que outras formas clínicas da micose possam ser observadas em crianças 8 . Aliás em 1982, Silva et al11 registraram um caso de lesão paracoccidióidica circunscrita ao pulmão, em uma menina de 14 anos de idade, procedente do Maranhão.

\section{AGRADECIMENTOS}

Ao Dr. Alberto Thomaz Londero (Professor Emérito, Universidade Federal de Santa Maria,
Santa Maria, RS), pelas críticas e sugestões.

\section{REFERÊNCIAS BIBLIOGRÁFICAS}

1. Andrade ALSS. Paracoccidioidomicose linfático-abdominal. Contribuição ao seu estudo. Revista de Patologia Tropical 12:165-256, 1983.

2. Azevedo PC. Algumas considerações sobre a blastomicose sul-americana e seu agente etiológico. Tese de Livre Docência, Faculdade de Odontologia, Universidade Federal do Pará, Belém, PA, 1954.

3. Campbell CC. "The pilot wheel": A change in course. Paracoccidioidomycosis. Proceedings of first Pan American Symposium. Pan American Health Organization. Scientific Publication no 254, Washington, p. 306-312, 1972.

4. Castro RM, Del Negro G. Particularidades clínicas da paracoccidioidomicose na criança. Revista do Hospital de Clínicas da Universidade de São Paulo 31:194-198, 1976.

5. Ferreira MS. Contribuição para o estudo clínicolaboratorial e terapêutico da formação juvenil da paracoccidioidomicose. Revista de Patologia Tropical 22:267-406, 1993.

6. Guimarães FN, Macedo DG. Contribuição ao estudo das blastomicoses na Amazônia (b. queloidiana e b. sulamericana). O Hospital 38:223-253, 1950.

7. Hildebrand TM, Rosário Filho NA, Queiroz Telles Filho F, Costa O, Miasaki, N, Mira JGS, Miyaki, M. Paracoccidioidomicose na criança. Aspectos clínicos e laboratoriais em 25 casos. Jornal de Pediatria 63:92-97, 1987.

8. Londero AT, Rios-Gonçalves AJ, Terra GMF. Paracoccidioidomycosis in Brazilian children. A critical review (1911-1994). Anais Brasileiros de Pediatria 4:128137, 1997.

9. Naiff RD, Barret TV, Arias JR, Naiff, MF. Encuesta epidemiologica de histoplasmosis, paracoccidioidomicosis y leishmaniasis mediante pruebas cutaneas. Boletin de la Oficina Sanitaria Panamericana 104:35-49, 1988.

10. Naiff RD, Ferreira LGL, Barret TV, Naiff, MF, Arias JR. Paracoccidioidomicose enzoótica em tatus (Dasypus novemcinctus) no Estado do Pará. Revista do Instituto de Medicina Tropical de São Paulo 28:19-27, 1986.

11. Silva JF, Tajra AD, Lima FC, Silva DB, Medeiros, MSR. Paracoccidioidomicose: registro de 38 casos observados em Teresina (PI). Revista Brasileira de Cirurgia 72:365367, 1982.

12. Veras KN. Paracoccidioidomicose. Estudo epidemiológico e clínico de pacientes internados no Hospital de Doenças Infecto-Contagiosas (HDIC) em Teresina (PI). Identificação de reservareas nos Estados do Pará e Maranhão. Dissertação de Mestrado em Medicina Tropical. Fundação Oswaldo Cruz, Universidade Federal do Piaui, Teresina, PI, 1995. 